\title{
La investigación y su importancia en el quehacer universitario
}

\author{
doi: 10.33264/rpa.201802-04
}

Gonzalo Araya Ibáñez

Escuela de Administración UNIACC

Facultad de Administración UNIACC

\section{Resumen}

En el presente artículo se presentan algunas reflexiones sobre la investigación universitaria y los beneficios que puede aportar al quehacer académico. Se revisa el último Informe Anual sobre el Comportamiento de las Instituciones de Educación Superior Iberoamericanas en materia de investigación, la importancia de la publicación científica y su impacto en la comunidad científica, lo que se complementa con información sobre la inversión chilena en investigación y desarrollo. También se analiza someramente las fuentes de financiamiento para la investigación y la preponderancia de los fondos públicos concursables. Finalmente, se le da una mirada a la investigación educacional y su impacto en los procesos de enseñanza aprendizaje.

Palabras clave: investigación, aprendizaje, gasto en I\&D, financiamiento.

\section{Abstract}

This article presents some reflections on university research and the benefits it can bring to academic work. The latest annual report on the behavior of Ibero-American higher education institutions regarding research is reviewed, as well as the importance of scientific publication and its impact on the scientific community, which is complemented by information on Chilean investment in research and development. The research funding sources and the preponderance of public competitive funds are also analyzed briefly. Finally, a look at educational research and its impact on teaching-learning processes is given.

Keywords: research, learning, investment in $R \& D$, funding sources 


\section{Introducción}

En un interesante seminario taller denominado "Igualdad de género y ciencia abierta en el escenario de investigación mundial" organizado por Elsevier, la mayor editorial de libros científicos del mundo, se presentó una muy sorprendente investigación sobre las diferencias de género en el campo de las publicaciones científicas a nivel mundial y de Chile en particular, además de una ponencia sobre Open Science (ciencia abierta), movimiento que busca la accesibilidad de las investigaciones científicas para todos los ciudadanos.

El estudio comparaba dos quinquenios separados por una década: 1996-2000 y 20112015, y los resultados mostraban, entre otros muchos datos de interés, que la participación de investigadoras chilenas con publicaciones científicas aumentó de 33\% en el primer quinquenio señalado a $38 \%$ en el segundo, un alza significativa pero que aún está lejos del $62 \%$ que muestran los investigadores nacionales en ese último período y lejos también de alcanzar un cincuenta por ciento que marcaría una igualdad total en esta materia. No obstante, estas cifras no están muy lejos de las que muestran EE.UU. (40\% y 60\%, respectivamente), Canadá (42\% y 58\%) y Australia (44\% y 56\%), algunos de los otros países considerados en el estudio. (Cid, 2018a)

También hay diferencias de género importantes en cuanto a las disciplinas en las que se realiza investigación en Chile. Por ejemplo, un 35\% de las investigaciones realizadas por mujeres se enfocan en el área de la medicina, genética y biología molecular, frente a un $26 \%$ de investigaciones realizadas por hombres en estas disciplinas. En ingeniería se invierte la relación, pues un $7 \%$ de las investigaciones realizadas por hombres se enfocan en esta materia, frente a un 3\% de las investigaciones lideradas por mujeres. (Cid, 2018a)

Por su parte, en la ponencia sobre Open Science se entregaron noticias alentadoras para los investigadores, pues cada vez más se están abriendo a nivel mundial los cerrados círculos por donde se transmite la investigación científica. De acuerdo con Cid (2018b), Suiza lidera a nivel mundial el acceso libre (open access) a artículos e investigaciones científicas, con un 39\% de artículos accesados de esa forma. En Chile, en tanto, el acceso vía open access a artículos científicos llega al 11\%.

Un ejemplo muy cercano de open access es el proyecto de alcance global llamado SciELO (Scientific Electronic Library Online o Biblioteca Científica Electrónica en Línea), que permite la publicación electrónica de ediciones completas de revistas científicas en una plataforma que posibilita el acceso libre a través de distintos mecanismos, incluyendo listas de títulos y por materia, índices de autores y materias 
y un motor de búsqueda. SciELO Chile (https://scielo.conicyt.cl/) se desarrolla con el apoyo de CONICYT, la Comisión Nacional de Investigación Científica y Tecnológica, dependiente del Ministerio de Educación.

Luego de este breve preámbulo, cabe preguntarse qué relación o interés puede tener la Universidad UNIACC con estos temas. Se estima que de variadas formas, según se explica en lo que sigue.

En primer lugar, los temas de género están muy de moda hoy en día y tienen un impacto transversal en toda la sociedad. Por supuesto, también en la academia. UNIACC, por lo demás, ha marcado un liderazgo en la materia, al contar con un cuerpo docente y de colaboradores mayoritariamente femenil. Baste señalar que de sus cuatro facultades, tres de ellas tienen a una Decana como su primera autoridad académica.

Asimismo, la referida diferencia de género en la publicación de investigación científica se mantiene en la Universidad, toda vez que en la última convocatoria al Concurso Anual de Investigación, año 2017, al que concursaron 12 proyectos de investigación, resultaron seleccionados finalmente 8 proyectos, 5 de los cuales eran liderados por investigadores varones y 3 por investigadores mujeres, manteniéndose curiosamente la misma relación en favor de los investigadores hombres (62,5\% frente a un $37,5 \%$ de investigaciones lideradas por mujeres).

En segundo término, entrando ya a temas concretos relacionados con la investigación -el objeto de este artículo- el dato que se menciona al comienzo de que las investigadoras tienen un cierto rezago respecto de sus pares masculinos en relación con las publicaciones científicas, da pie para mirar en qué posición se encuentran las universidades chilenas en cuanto a ese importante rubro de su quehacer académico.

El último Informe anual sobre el Comportamiento de las Instituciones de Educación Superior Iberoamericanas en materia de investigación (SIR Iber 2018), publicado por SCImago Research Group, ofrece una clasificación según el número de trabajos publicados en Scopus en el período 2012-2016 y analiza su desempeño en base a tres factores fundamentales: Investigación, Innovación e Impacto social.

Un paréntesis, que muestra que todo lo dicho previamente está relacionado de una manera u otra. Scopus es una base de datos bibliográfica de resúmenes y citas de artículos de revistas científicas, perteneciente a Elsevier, la editorial citada al comienzo. En el SIR Iber se incluyen instituciones de educación superior que tengan al menos un documento publicado en revistas indexadas en Scopus a lo largo del 
quinquenio analizado. Cabe señalar que existe también el índice SIR World, donde se incluyen instituciones de todos los países y sectores que hayan publicado como mínimo 100 documentos en revistas indexadas en Scopus en el último año del período de estudio.

En la siguiente tabla se muestran algunos indicadores utilizados en el referido Informe, para las 10 primeras universidades del país:

Tabla $N^{\circ}$ 1. Clasificación universidades chilenas en el SIR Iber 2018

\begin{tabular}{|c|c|c|c|c|c|}
\hline \multirow{2}{*}{ Institución } & \multicolumn{3}{|c|}{ Ubicación Informe 2018} & \multirow{2}{*}{ Producción } & \multirow{2}{*}{$\begin{array}{c}\text { Impacto } \\
\text { normalizado }\end{array}$} \\
\hline & Nacional & LA & Ibero & & \\
\hline U. de Chile & 1 & 10 & 24 & 12.616 & 0,86 \\
\hline U. Católica & 2 & 12 & 29 & 10.975 & 0,73 \\
\hline U. de Concepción & 3 & 30 & 61 & 5.264 & 0,67 \\
\hline USACH & 4 & 57 & 106 & 2.902 & 0.61 \\
\hline UTFSM & 5 & 59 & 111 & 2.809 & 1,09 \\
\hline UC Valparaíso & 6 & 64 & 118 & 2.543 & 0,70 \\
\hline U. Austral & 7 & 67 & 121 & 2.480 & 0.70 \\
\hline U. Andrés Bello & 8 & 71 & 127 & 2.334 & 0,74 \\
\hline U. de la Frontera & 9 & 85 & 143 & 2.012 & 0,55 \\
\hline U. de Valparaíso & 10 & 91 & 149 & 1.826 & 0,56 \\
\hline
\end{tabular}

Nota: Elaboración propia, con información de SIR Iber 2018

El indicador de producción representa el número total de documentos publicados por la Institución en revistas indexadas en Scopus, en tanto que el indicador de impacto normalizado, refleja el impacto del conocimiento generado por una institución en la comunidad científica internacional.

Los valores que se muestran en la última columna toman como punto central la media mundial de impacto (a la que se le asigna el valor 1). Por consiguiente, si una institución tiene un impacto normalizado de 0,86, como es el caso de la U. de Chile, significa que su producción se cita un $14 \%$ por debajo del promedio mundial. Por el contrario, si una institución tiene un impacto normalizado de 1,09 (como sucede con la Universidad Técnica Federico Santa María), significa que su producción se cita un $9 \%$ por encima del promedio mundial de citación.

Cabe señalar que este informe utiliza además otros parámetros muy interesantes para realizar su clasificación, entre los que destacan los indicadores Excellence with Leadership (EwL) que refleja el porcentaje de producción de una institución que se encuentra dentro del 10\% de trabajos más citados en su categoría de conocimiento, y 
Scientific Talent Pool (STP), que representa el número de autores diferentes de una misma institución que han participado en el total de trabajos publicados. Todos los parámetros citados, que se relacionan con el factor investigación, se obtienen de Scopus. La innovación, que se mide a través de indicadores relacionados con la obtención de patentes, utiliza como fuente la Oficina de Patentes Europea (www.epo.org) en tanto que el impacto social se mide mediante Google y Ahrefs (https://ahrefs.com/).

Como dato anexo, se puede señalar que UNIACC aparece en dicho Informe, en la posición 52 a nivel nacional y compartiendo la posición 588 en Iberoamérica, con una producción de 5 trabajos, lo que representa un gran desafío de cara al futuro, teniendo en consideración que dichos trabajos fueron presentados entre los años 2012 y 2013.

De aquello se puede colegir que si la Revista Pensamiento Académico se indexa a Scopus, con seguridad la posición de UNIACC en el ranking SIR Iber -y en general, en todo otro informe similar- comenzará a subir significativamente, pues aquella es una de las plataformas idóneas para la publicación de las investigaciones que se llevan a cabo en la Universidad.

Ahora bien, es importante señalar que existe consenso que uno de los indicadores bibliométricos más aceptados para analizar el desempeño de la producción científica es la citación, considerada como una muestra del reconocimiento de trabajos previos y una expresión de la influencia del autor que es citado sobre el autor que realiza la cita (SIR Iber, 2018).

No obstante ello, la evolución de la bibliometría ha llevado al desarrollado de otros indicadores relacionados con producción científica y con otros tipos de resultados de investigación, de modo que cada vez cobran más importancia factores como el impacto social (visibilidad web) y la innovación en la generación de conocimiento.

Por consiguiente, al unir los enfoques de producción científica, de innovación y de impacto social, como lo hace el informe anual de por SCImago Research Group, se puede contar con una evaluación de carácter integral y con componentes que enriquecen la medición del impacto y hacen visibles nuevos usos de la información (SIR Iber 2018).

Para concluir este tema, es relevante mencionar que de dicho informe anual se puede colegir que las universidades chilenas producen el $89,98 \%$ de la ciencia nacional. Una cifra impresionante, que demuestra la importancia de la investigación dentro de las 
universidades y su impacto a nivel nacional. Hay que recordar sobre este punto que hay un importante incentivo tributario para la investigación y desarrollo, que fomenta precisamente la interacción entre los privados y las universidades en esta materia, de modo que la investigación sirva a propósitos más prácticos (investigación aplicada), potenciando directamente las fuentes productivas del país.

\section{Algunos datos sobre inversión en investigación y sus fuentes de financiamiento}

No es un misterio que la investigación es clave en el desarrollo de los países. Ya lo decía el economista estadounidense Gary Becker, Premio Nobel de Economía del año 1992 por sus trabajos sobre comportamiento humano, cuando expresaba -hace ya muchos años- que "el éxito o el fracaso económico de una nación ya no depende de la tecnología o de la maquinaria que posee, sino de lo que haga con su gente". Y la investigación tiene mucho que decir al respecto, pues sus efectos se hacen sentir en el progreso y el bienestar de las personas (Peña, 2016).

Empero, nuestro país no invierte mucho en investigación y desarrollo (I+D). Una de las últimas cifras que se manejan al respecto (año 2015) señala que Chile invierte sólo el equivalente al $\mathbf{0 , 3 9 \%}$ del PIB en investigación y desarrollo, la cifra más baja de todos los países miembros de la Organización para la Cooperación y el Desarrollo Económicos (OCDE), cuyo promedio está en $\mathbf{2 , 3 8 \%}$ del PIB. Y aquello no es un dato menor, pues esos guarismos muestran la preocupación de los Estados por la investigación (Emol.com).

Al observar las cifras provisionales que maneja dicho Organismo, se puede apreciar que para el año 2016 la inversión en I+D fue incluso menor (0,36\% del PIB), lo que muestra un rezago importante en la materia, incluso respecto de los países vecinos, pues Argentina, por ejemplo, ha invertido un 0,53\% de su PIB en I+D en ese mismo año (OCDE, 2018).

En el gráfico siguiente, que muestra datos del año 2013 (o del año que se indica expresamente en algunos casos), se puede apreciar lo distante que estamos de los países de la OCDE y de algunos otros seleccionados que se incluyen en la gráfica.

Israel y Corea, por ejemplo, que lideran esta muestra, muestran cifras que representan más de 10 veces de lo que invierte Chile en I+D, medido como porcentaje de los respectivos PIB de cada país. Una brecha que impresiona. 
Gráfico N 1. Gasto en I+D como porcentaje del PIB. Año 2013

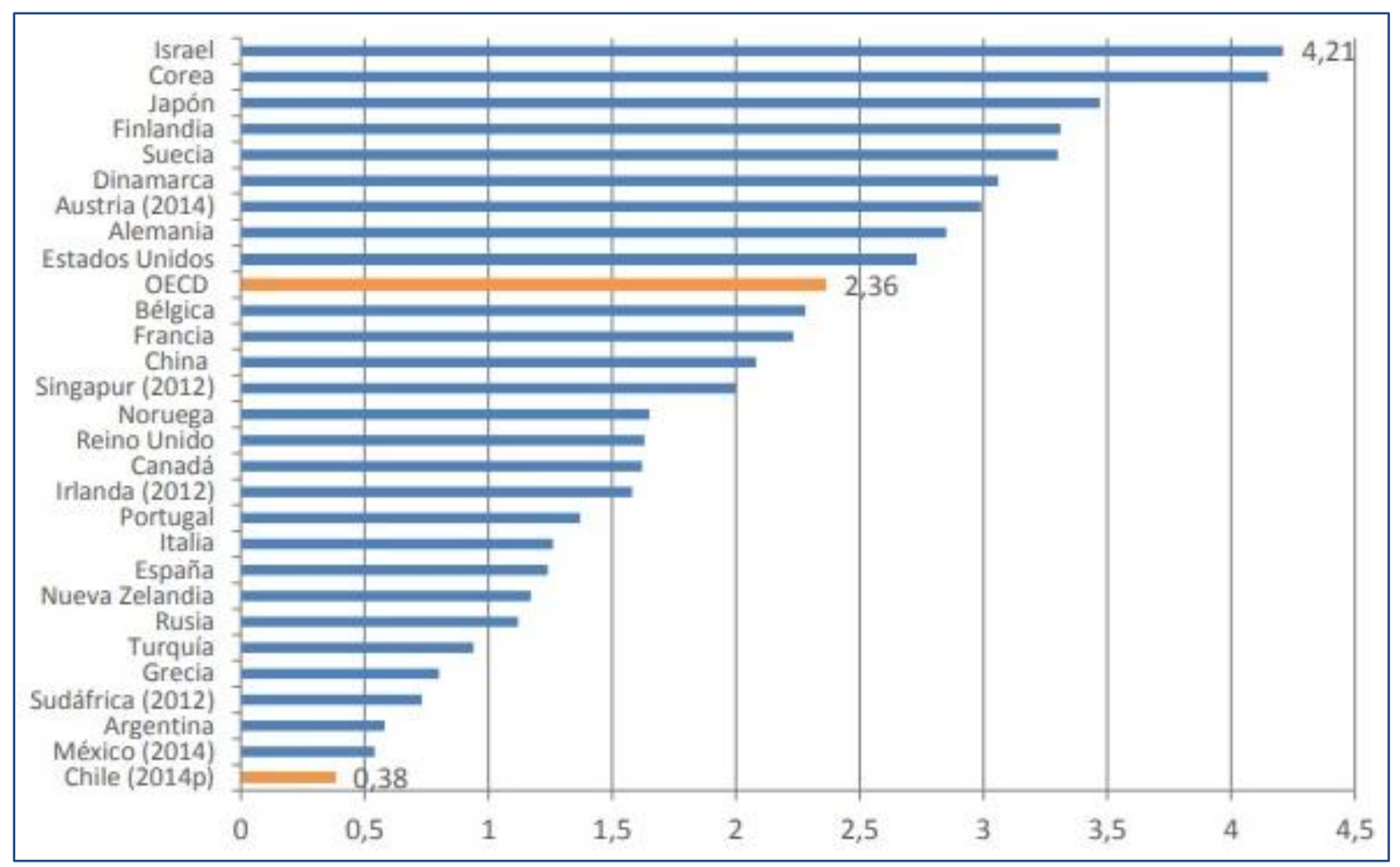

Nota: Tomado de Rodríguez (2016, p. 3)

Nótese que los valores no cambiaron casi nada en las dos mediciones. Chile subió de 0,38\% a 0,39\% y el promedio de la OCDE se elevó de 2,36\% a 2,38\% en el año 2015. Lo preocupante es que la posición de Chile respecto de los países latinoamericanos se ha debilitado, pues Brasil, Argentina y México invierten más en I+D que nosotros (Rodríguez, 2016).

Otro dato desalentador es que, en términos del capital humano, el número de investigadores chilenos por cada 1.000 habitantes (en jornadas completas equivalentes) es el más bajo de la OCDE. En efecto, considerando las cifras provisionales para el año 2016, en el país existe un investigador cada 1.000 personas. La cifra exacta que indica la OCDE es de 1,04 por mil, que si bien ha ido subiendo desde un magro 0,7 por mil, en el año 2010, es claramente muy baja, respecto del promedio de la OCDE (7,74 por mil) o de Dinamarca, país que presenta el índice más alto, llegando a 14,33 investigadores por cada mil habitantes (OCDE, 2018).

Pero no todo es tan oscuro, pues la mayor parte de la ejecución del gasto en I+D corre por cuenta de las universidades. Y Rodríguez (2016) aporta datos muy interesantes al respecto. Por ejemplo, que la estructura del gasto es muy similar en las instituciones estatales y privadas, con un fuerte predominio del gasto en remunerar a los investigadores, lo que se acentúa mucho más en las entidades privadas, como puede apreciarse en el Gráfico $\mathrm{N}^{\circ} 2$. 
Gráfico $N^{\circ}$ 2. Desglose del gasto en I+D (en millones de $\$$ del año 2014)

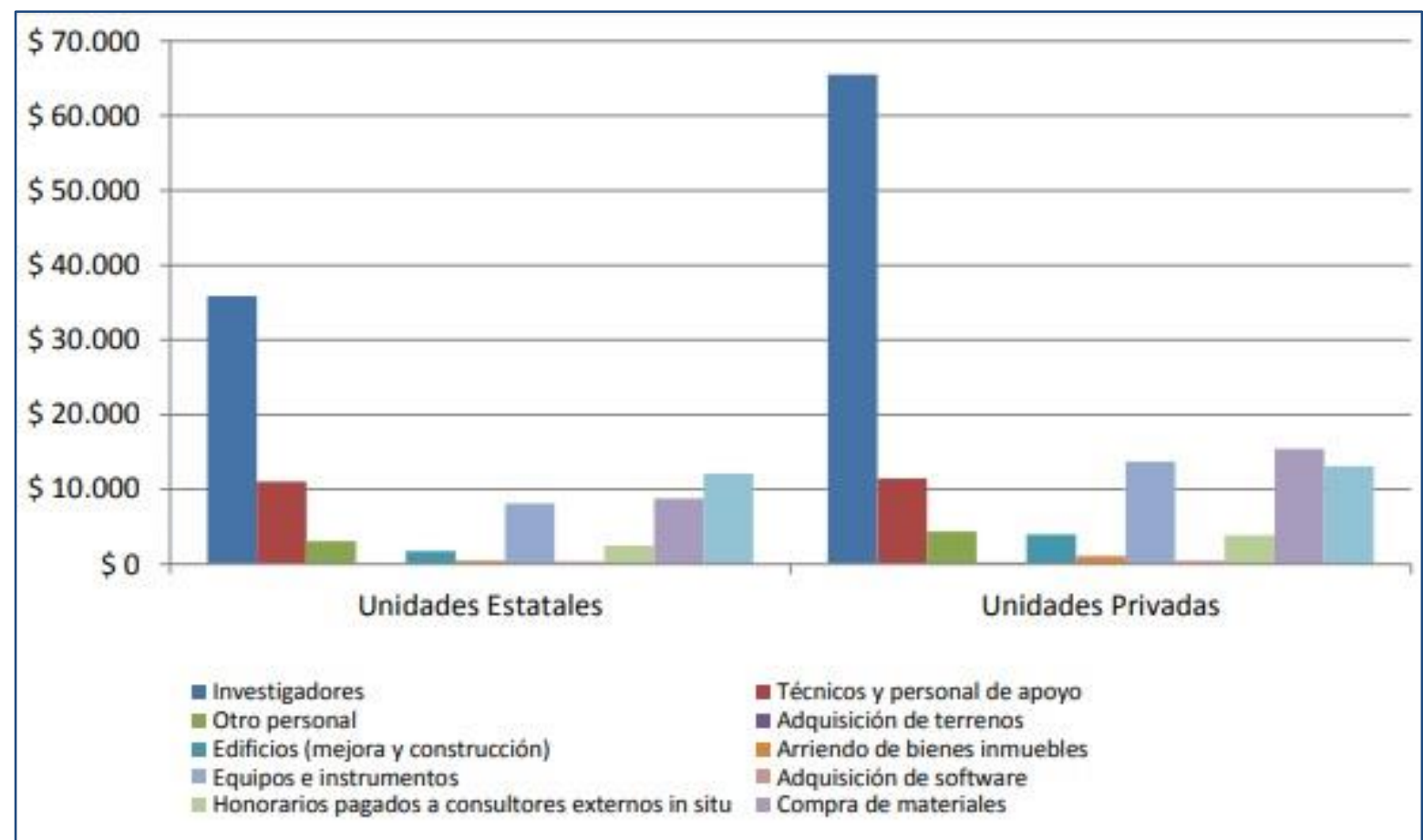

Nota: Tomado de Rodríguez (2016, p. 6)

Aquello hay que relacionarlo con las fuentes de financiamiento. El gráfico siguiente muestra dicha información, destacando que los fondos públicos concursables son los que priman como fuente generadora de recursos, representando el $55 \%$ de los fondos (Rodríguez, 2016).

Gráfico $\mathbf{N}^{\circ}$ 3. Fuentes de financiamiento de l+D ejecutado por universidades (En millones de pesos del año 2014)

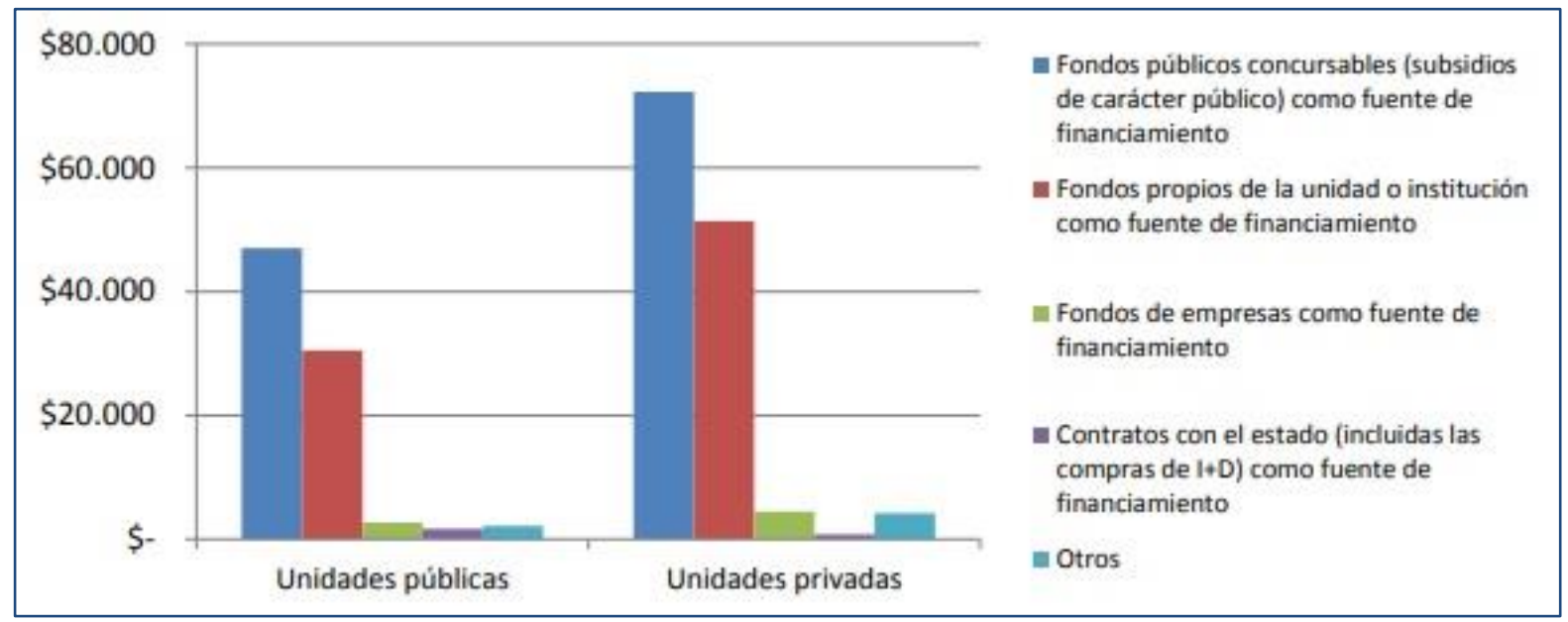

Nota: Tomado de Rodríguez (2016, p. 9) 
Es necesario señalar que la mayor parte de los fondos públicos concursables provienen de CONICYT, ente gubernamental que mantiene varios instrumentos de financiamiento de proyectos de investigación, como el FONDECYT (Fondo Nacional de Desarrollo Científico y Tecnológico), concurso que actualmente está abierto en su modalidad regular para el año 2019.

Este instrumento financia exclusivamente proyectos de investigación científica o tecnológica, esto es, que conduzcan a nuevos conocimientos o aplicaciones. No financia proyectos de creación artística, recopilaciones, confección de catálogos o inventarios, impresión de libros, ensayos, traducciones, audiovisuales, textos de enseñanza u otras actividades análogas. Requiere patrocinio de instituciones con personería jurídica en Chile, tales como universidades y otras entidades de educación superior.

También es relevante mencionar que CONICYT financia la formación de investigadores chilenos a nivel de magíster y doctorado mediante becas, lo que es clave en el desarrollo de la investigación en una universidad, dado que especialmente los doctores- son productores de investigación original, la que hará parte de la producción atribuible a la universidad lo que a su vez sumará para escalar en los diversos rankings (como el SIR Iber), a lo que se puede agregar el apoyo que pueden prestar en líneas de investigación de los docentes ya presentes en las universidades, expandiendo y fortaleciendo la capacidad investigativa instalada (Rodríguez, 2016).

Para finalizar esta parte, destacar una de las conclusiones del investigar recién citado: Chile debiera contar con instrumentos de financiamiento de la investigación a nivel institucional, y no exclusivamente a nivel de investigadores y proyectos, como ocurre en la actualidad. Aquello sería sin duda un impulso para el desarrollo de la investigación en universidades que no cuentan con un perfil investigativo claro ni mucha historia relacionada con este interesante tema, como es el caso de la Universidad UNIACC.

Con seguridad este será uno de los principales desafíos del nuevo Ministerio de Ciencia, Tecnología, Conocimiento e Innovación, que tiene por misión asesorar y colaborar con el Presidente de la República en el diseño, formulación, coordinación, implementación y evaluación de las políticas, planes y programas destinados a fomentar y fortalecer la ciencia, la tecnología y la innovación derivada de la investigación científico-tecnológica. 


\section{Rol de la investigación dentro de las instituciones de educación superior}

La investigación científica sirve sin duda para generar nuevos conocimientos, adelantos tecnológicos, mejoras en la calidad de vida y un sinnúmero de otros beneficios de interés general. En el imaginario colectivo, el investigador científico es visto como un genio loco o un Giro Sintornillos (la caricatura de Disney) que siempre está creando e inventando; pero la investigación no siempre está llamada a estas grandes batallas. También es muy importante a pequeñas escalas, mejorando procesos, haciéndolos más eficientes, resolviendo pequeños problemas que, sumados, pueden hacer grandes cambios.

No puede perderse de vista que casi el 90\% de toda la investigación científica en Chile se desarrolla en las Universidades, como se mencionó precedentemente.

Igual de importante para las universidades es también el proceso mismo de investigación, la rigurosidad en la aplicación del método científico, los caminos que se abren al investigar, la motivación que los investigadores pueden incubar en los demás docentes, el mejoramiento en los ranking que la investigación conlleva y el círculo virtuoso que se genera con todo ello, que incluye una mayor atracción de alumnos y de fondos para investigar.

Desde el punto de vista de la formación, no cabe duda que la investigación es clave para los procesos de enseñanza-aprendizaje. Así por lo demás lo ha entendido la Universidad UNIACC al convocar a los concursos anuales de investigación, que actualmente va en su tercera versión, con focos puestos en la educación y el mejoramiento de la docencia.

En efecto, la investigación en educación debe ser vista como un proceso indispensable para renovar y transformar los ambientes de enseñanza y aprendizaje, logrando calidad en la educación, vale decir, que responda a las necesidades de los estudiantes según sus contextos. (Muñoz y Garay, 2015)

Asimismo, de acuerdo con Sierra (2004), "la investigación y la docencia deben conformar una unidad de acción para el investigador, ya que es ésta la mejor manera de aportar al estudiante contenidos que eleven el nivel académico", agregando que dicha unidad permite al docente reflexionar sobre sus inquietudes intelectuales y científicas en la medida en que investiga y traspasa parte de esas inquietudes y conocimientos a un auditorio más ávido y preparado.

La investigación proporciona nuevas y mejores formas de comprensión sobre la dinámica de los procesos de enseñanza y aprendizaje, de modo que la mejora de los 
procesos y resultados educativos debe ser el principal propósito de la investigación educativa (Muñoz y Garay, 2015).

En este punto, quizás convenga hacer un guiño al desarrollo curricular, fundamentado en la praxis, rescatando la práctica como aspecto relevante del quehacer universitario. En este enfoque, algunos teóricos han planteado la idea de desarrollar una cultura de investigación en la que los docentes ocupen un papel central en la determinación de los fines educativos, no sólo con el propósito de ofrecer soluciones, sino que resolver problemas prácticos derivados de la realidad de la institución que los acoge (Abraham, 2000).

Así entonces, el docente-investigador no sólo deberá aplicar una secuencia de rutinas preestablecidas, sino que está llamado a crear una manera personal de intervención a partir del diagnóstico y evaluación continua de la situación del aula (Abraham, 2000).

En este orden de ideas, una herramienta que parece adaptarse muy bien a este contexto es la investigación-acción, cuya finalidad esencial, de acuerdo con Sandín (2003), citando a Elliott, unos de los padres de esta metodología, "no es la acumulación de conocimientos sobre la enseñanza o la comprensión de la realidad educativa, sino que, fundamentalmente, aportar información que guíe la toma de decisiones y los procesos de cambio para la mejora de la misma” (p. 161).

Una metodología que sin lugar a dudas debería aplicarse con mayor asiduidad en la Universidad pues precisamente el objetivo prioritario de la investigación-acción consiste en mejorar la práctica docente en vez de generar conocimientos, de modo que la producción y utilización del conocimiento se subordina a dicho objetivo fundamental y queda, por tanto, condicionado por él (Sandín, 2003).

Como no es la intención profundizar más sobre este concepto, que importa una reflexión sistemática sobre las prácticas docentes, sólo se mencionarán algunas de las áreas de aplicación de la investigación-acción, que ayudarán a comprender su importancia para los procesos de enseñanza-aprendizaje: i) desarrollo curricular, ii) introducción de nuevas estrategias de enseñanza-aprendizaje, iii) innovación educativa, iv) cambio de actitudes, v) formación permanente del cuerpo docente.

Con investigación-acción se pueden identificar y resolver problemas que dificulten el desenvolvimiento del docente en su aula y que por lo tanto pongan trabas al aprendizaje de los alumnos, mediante un proceso iterativo que consta de cuatro fases: i) Diagnóstico y reconocimiento de la situación inicial; ii) Desarrollo de un plan de acción, críticamente informado, para mejorar aquello que ya está ocurriendo; iii) 
Actuación para poner el plan en práctica y la observación de sus efectos en el contexto que tiene lugar, y iv) La reflexión en torno a los efectos como base para una nueva planificación (Bausela, s.f., citando a Kemmis McTaggart, 1988).

Terminado ese breve paréntesis, puede ser también muy relevante para las instituciones de educación superior traspasar las bondades de la investigación a sus propios alumnos. Hacer del alumno un investigador, crítico, autogestor, generador de desarrollo, capaz de interactuar y trabajar en equipo y de proponer alternativas en pos de cumplir con sus objetivos propuestos, harán más por su desarrollo personal y profesional que otro tipo de intervenciones. (Peña, 2016)

$\mathrm{Y}$ es porque precisamente la Universidad es un espacio para fomentar estas capacidades; un lugar ideal para investigar, innovar, desarrollar el espíritu creativo. El "bienvenido a crear" de la Universidad UNIACC adquiere un sentido mucho más amplio cuando se le piensa desde la óptica de la investigación.

No se puede perder de vista que cuando los estudiantes logran desarrollar estas habilidades, que implican además la puesta en práctica de los aprendizajes adquiridos, se enriquece tanto su formación como el proceso general de enseñanzaaprendizaje, haciéndose éste mucho más dinámico.

\section{Referencias}

Abraham, M. (2000). Análisis de algunas perspectivas del currículum. Magíster en Educación Superior, UNIACC.

Bausela, E. (s.f.). La docencia a través de la investigación-acción. Universidad de León, España. Recuperado el 28 de diciembre de 2018 de: https://rieoei.org/historico/deloslectores/682Bausela.pdf

Cid, D. (Agosto, 2018a). El equilibrio de géneros en la investigación científica. En Igualdad de género y ciencia abierta en el escenario de investigación mundial. Seminario llevado a cabo por Elsevier, Santiago.

Cid, D. (Agosto, 2018b). Enabling open science. En Igualdad de género y ciencia abierta en el escenario de investigación mundial. Seminario llevado a cabo por Elsevier, Santiago.

De Moya, F.; Herrán, E.; Bustos, A.; Corera, E.; Tibaná, G. (2018). Ranking Iberoamericano de instituciones de educación superior. SIR Iber 2018. Barcelona: Ediciones Profesionales de la Información SL. 
Emol (30 de enero de 2017). Inversión en I+D en Chile sube 4,4\% pero aún es la más baja de los países OCDE. Emol. Recuperado el 31 de agosto de 2018 de: http://www.emol.com/noticias/ Economia/2017/01/30/842436/Inversion-en-ID-enChile-sube-44-pero-todavia-es-la-mas-baja-de-los-paises-OCDE.html

Muñoz, M., \& Garay, F. (2015). La investigación como forma de desarrollo profesional docente: Retos y perspectivas. Estudios pedagógicos (Valdivia), 41(2), 389-399. doi: 10.4067/S0718-07052015000200023

Organización para la Cooperación y el Desarrollo Económicos (2018). Main Science and Technology Indicators 2018. Recuperado el 27 de diciembre de 2018 de http://www.oecd.org/sti/msti.htm

Peña, C. (2016). La importancia de la investigación universitaria y de incorporar la misma al proceso formativo. Revista electrónica Click, Universidad de Aquino, Bolivia. Recuperado el 29 de agosto de 2018 de http://www.udabol.edu.bo/blog/laimportancia-de-la-investigacion-universitaria-y-de-incorporar-la-misma-en-elproceso-formativo/

Rodríguez, D. (2016). Financiamiento de la investigación en Chile. Revista AcciónEducar, 3(41). Recuperado el 27 de diciembre de 2018 de: http://accioneducar.cl/wp-content/uploads/2016/08/Financiamiento-de-lainvestigación-universitaria-en-Chile.pdf

Sandín, M. (2003). Investigación Cualitativa en Educación: Fundamentos y tradiciones. Barcelona: McGraw-Hill.

Sierra, J. (2004). La investigación como prioridad universitaria. Revista Virtual Universidad Católica del Norte, 1(12). Recuperado el 27 de diciembre de http://revistavirtual.ucn.edu.co/index.php/RevistaUCN/article/view/285/540

\section{Gonzalo Araya Ibáñez}

Ingeniero Civil Industrial de la U. de Chile. MBA UNIACC. Diplomados Analista Tributario (USACH) y de Estrategias Tributarias. Magíster en Educación Superior UNIACC (en curso). Docente e Investigador de la UNIACC. Consultor y asesor tributario enfocado en empresas de menor tamaño. Autor de más de 15 obras en materias de gestión e impuestos. Actualizador del CheckPoint Tributario de Thomson Reuters, el sistema de información tributario líder del mercado. Colaborador permanente del Manual de Consultas Tributarias, con numerosos artículos publicados. Asesor Económico de Insolvencias. 